\title{
The evaluation of nerve damage in leprosy
}

\author{
J M H PEARSON \\ Dhoolpet Leprosy Research Centre, Hyderabad 500 006, India
}

Received for publication 29 October 1981

\section{Introduction}

Nerve damage is an almost invariable accompaniment of leprosy. It can increase even under chemotherapy; this is a significant risk for patients who develop reactions. Nerve damage may recover partially or completely with effective treatment.

The assessment of nerve damage is of the first importance in evaluating the results of therapy of neuritis, whether chemotherapy, anti-inflammatory therapy, or nerve surgery. The availability of corticosteroids and other drugs to control neuritis, and the present interest in surgical treatment of neuritis, make it important that any therapeutic procedure is evaluated in the short and long term by the most precise methods that can, in practice, be employed at each treatment centre.

The aim of this paper is to present a protocol with a limited objective, namely to define and describe in detail tests which could be used to evaluate the progress (deterioration or recovery) of damage to the ulnar and median nerves. These tests, performed, recorded, and graded as described, and repeated periodically, will be of value in enabling different centres to report the results of their studies in a standard and comparable format. Though planned with particular reference to nerve damage caused by leprosy, it is possible that they will be of use in assessing the treatment of injuries to the median and ulnar nerves.

The tests are in general simple to perform; most of them could be undertaken by a person without professional qualifications, trained specifically for this task. They should, however, be performed by staff not directly concerned with the treatment of the patient and who are not aware of the results of previous tests. This will help to eliminate bias in favour of an expected or hoped-for result.

The frequency of testing will vary according to whether the patient is in 
hospital or an outpatient, and whether time and staff are freely available. As a minimum they should be undertaken: 1 , at the start of the study; 2,1 day before an 'important event', e.g. surgical treatment or commencement of new drug therapy; 3, 2 weeks post-operation/2 weeks in trial; 4, 4 weeks; 5, 2 months; 6 , after 3 months; 7, after 6 months; and 8, 6 monthly thereafter. During the periods between full assessments it would be sound practice for a single sensory test and a single motor function test to be undertaken very frequently; definite improvement or deterioration of either test will indicate the need for full assessment to be repeated.

A scoring system has been suggested, so that progress or deterioration of nerve damage can be expressed quantitatively. This system is arbitrary; it has, however, been designed to give appropriate 'weight' to the results of different tests. In particular, greater weight is attached to the sensory tests as a whole than to the motor function tests, because the latter may improve due to muscle hypertrophy in the absence of improved nerve function. Also, results of tests (such as nerve tenderness) where there is a large subjective element carry less weight than the more objective tests.

Many leprosy centres concerned with the treatment of neuritis have competent staff but severe limitations on time and facilities. It is hoped that this protocol will encourage such centres to undertake planned trials of the treatment of neuritis, using tests that are within their means.

Neuritis is too important a subject to be studied in any but a well-conceived, well-thought-out protocol. This document is not such a protocol; it merely gives tests which are suitable for evaluating the neurological results of treatment. Those planning trials of the treatment (medical or surgical) of neuritis must ensure that the trial design is sound, and that sufficient numbers of patients will be included to obtain statistically valid results.

\section{Protocol for evaluating the treatment of nerve damage affecting the ulnar and median nerves}

\section{SUMMARY OF TESTS}

The tests employed indicate sensory and motor functions, as well as recording pain, tenderness and the possible presence of constrictions of the ulnar nerve in the region of the elbow. The sensory tests are derived from current understanding of the neurophysiological characteristics of cutaneous receptors in the glabrous skin of the hand: however, awareness that distal cutaneous nerve branches and endings can be damaged in leprosy is essential to the interpretation of the results of tests of the function of larger, more proximal nerves. These tests and their scores are summarized in Tables 1 and 2. 
Table 1. Summary of tests in protocol

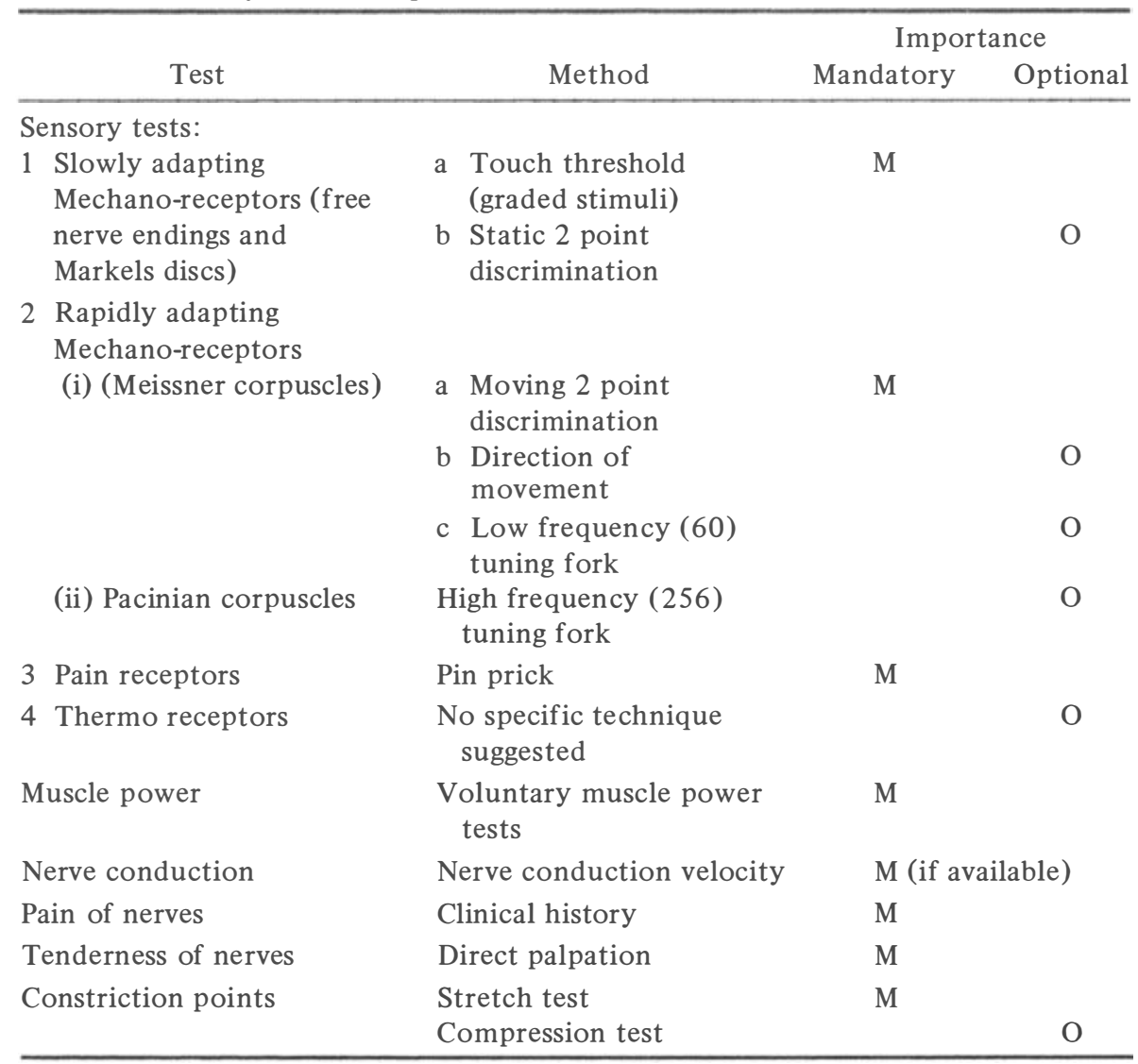

Table 2. Summary of scores

\begin{tabular}{lcc}
\hline \multicolumn{1}{c}{ Test } & \multicolumn{2}{c}{ Maximal score per nerve } \\
& Mandatory & Optional \\
\hline Touch threshold & 12 & - \\
Static two-point discrimination & - & 9 \\
Moving two-point discrimination & 9 & - \\
Direction of movement & - & 9 \\
Tuning fork - Low frequency & - & 3 \\
Tuning fork - High frequency & - & 3 \\
Pin prick & 9 & - \\
$\quad$ Totals for sensory tests & 30 & 24 \\
Voluntary muscle power & 10 & - \\
Nerve conduction velocity & 20 & - \\
Nerve pain & 3 & - \\
Nerve tenderness & 3 & - \\
Stretch test & 3 & - \\
Compression test & - & 3 \\
$\quad$ Totals & 69 & 27 \\
\hline
\end{tabular}




\section{PRINCIPLES OF SENSORY TESTING}

1 Do not go on for too long; $20 \mathrm{~min}$ is long enough for most patients. If necessary, do tests in two or more sessions.

2 Conduct tests in a quiet and comfortable room with minimal possible extraneous disturbance.

3 All tests are performed with the patient blindfolded (or with a barrier so he cannot see the stimulus) but always demonstrate the test before blindfolding, using a skin area with normal sensitivity. Ensure that the patient understands what is required of him before starting the recorded tests.

4 Always supply, from time to time, stimuli that will be felt (if necessary by stimulating an irrelevant site). Every third or fourth stimulus should be one which the patient can feel.

5 All tests should be carried out with the arm and hand relaxed, supinated and supported to the finger tips on a soft surface. This will prevent positive response due to awareness of movement of the fingers being recorded as, for instance, sensitivity to touch.

HOW TO PERFORM TESTS

Sensory tests

For all tests, 6 areas each about $1 \mathrm{~cm}$ diameter should be tested (see Fig. 1)

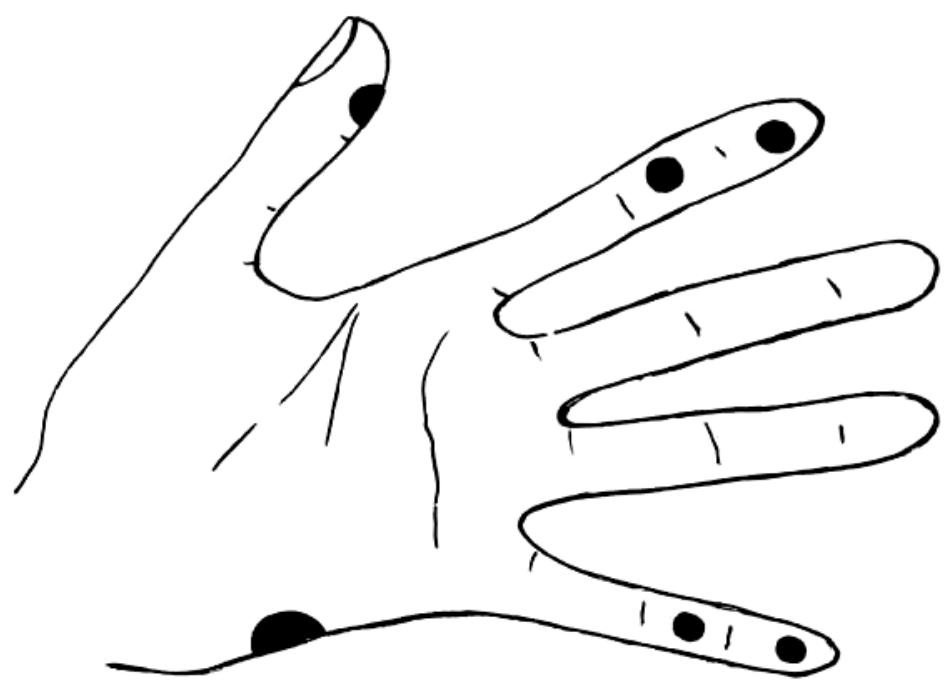

Figure 1. Areas where stimuli for sensory tests are applied. 
Ulnar supplied

1 Distal pulp, little finger

2 Proximal phalanx, little finger

3 Ulnar border of hand.

Median supplied

1 Distal pulp, index finger

2 Proximal phalanx, index finger

3 Distal pulp, thumb.

Within these areas, sites where the skin is abnormal (where, for instance, callus or scars are present) should be avoided for testing purposes.

Touch threshold

The stimulator (Fig. 2) consists of a set of 4 nylon threads mounted on wire handles. The threads are of different lengths, calibrated to bend slightly when forces of $0.5 \mathrm{~g}, 2.0 \mathrm{~g}, 5.0 \mathrm{~g}$, and $10.0 \mathrm{~g}$, respectively are applied. Details of their fabrication are given in the Appendix.

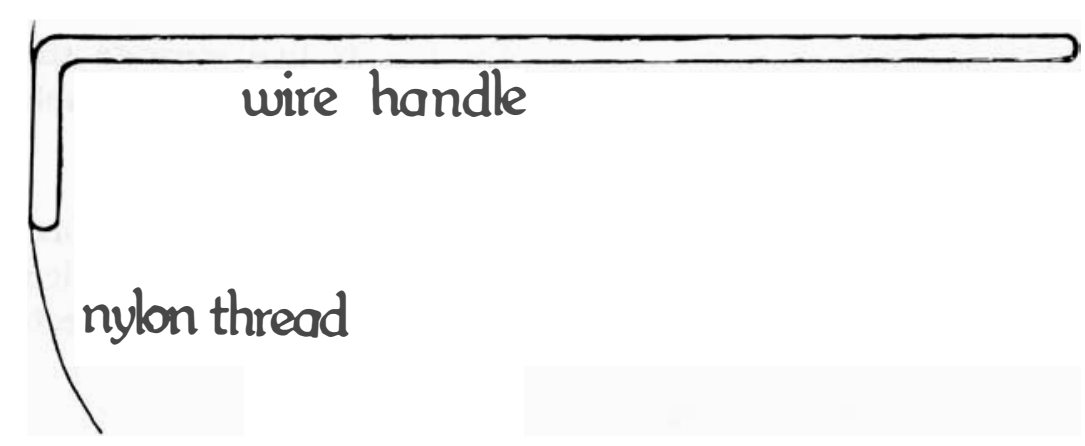

Figure 2. Stimulator for testing light touch threshold.

Method. The thread is touched once lightly on the skin at the test sites, and then withdrawn (as if testing for pin prick). The force should be sufficient to bend the thread slightly. One to three individual stimuli are delivered in each area (sufficient to be confident whether the thread is felt or not felt). It is best for the test sites to be stimulated in random order, each area being touched only once at a time; the examiner should return to a site after testing another site if there was doubt the first time. A single site should not be touched several times in quick succession. The patient, whose eyes are closed or covered, touches the point where he feels the thread; he is not informed of the moment when the stimulus is delivered.

Scoring. The softest touch consistently felt in each area will be recorded, and scored as in Table 3 . For each nerve, 3 sites are stimulated. The maximum possible score for each nerve is therefore 12 points. 
Table 3. Scoring for touch threshold

\begin{tabular}{cc}
\hline $\begin{array}{c}\text { Force applied by softest } \\
\text { bristle felt (gn) }\end{array}$ & Score \\
\hline 0.5 & 4 \\
2 & 3 \\
5 & 2 \\
10 & 1 \\
No bristle felt & 0 \\
\hline
\end{tabular}

Static two-point discrimination test

The stimulating object is the two blunt ends of a paperclip, or the legs of a blunt caliper. Stimuli should be applied with the legs 3, 6 and $12 \mathrm{~mm}$ apart. The caliper must be applied exactly vertical to the skin, with just sufficient pressure to blanche the underlying skin, and the pressure maintained for exactly $2 \mathrm{~s}$ before the caliper is removed. The patient must state whether he feels one touch or two. One to three stimuli are delivered in each area (sufficient to be confident of the result).

Scoring. The shortest distance consistently felt as two separate stimuli is noted, and scored for each site as in Table 4. The maximum possible score for one nerve is 9 points.

Note. There is considerable observer variation in the results of this test careful training is required. In particular, because skin affected by leprosy is often abnormal, the correct pressure must be learnt using normal subjects.

Table 4. Scoring for static and moving twopoint discrimination tests

\begin{tabular}{cc}
\hline $\begin{array}{c}\text { Distance between legs } \\
\text { of caliper }(\mathrm{mm})\end{array}$ & Score \\
\hline 3 & 3 \\
6 & 2 \\
12 & 1 \\
Not felt or always & 0 \\
felt as one touch & \\
\hline
\end{tabular}

Moving two-point discrimination test

Use the same stimulating object(s), as for the static test. Apply the caliper lightly to the distal part of the test area and draw it gently $1 \mathrm{~cm}$ proximally. Perform the test one to three times in each area (sufficient to be confident of the result). Record the shortest distance between the caliper legs that is consistently felt as two stimuli, and score as in Table 4. The maximum score for one nerve is 9 points. 
Table 5. Scoring for direction of movement test

\begin{tabular}{cc}
\hline Distance moved $(\mathrm{mm})$ & Score \\
\hline $0-3$ & 3 \\
$4-6$ & 2 \\
$7-12$ & 1 \\
No movement felt or & 0 \\
no stimulus felt & \\
\hline
\end{tabular}

\section{Direction of movement test}

Draw a blunt metal probe lightly along the area to be tested, either distoproximally or proximo-distally. Record the distance moved at which the patient appreciates the direction of movement. Perform the test one to three times in each area (sufficient to be confident of the result). Score as in Table 5. The maximum score for one nerve is 9 points.

Tuning-fork tests

Any pattern of tuning fork may be used for these tests: it should be brought into resonance by tapping one arm firmly on the knee (hard surfaces damage the instrument). For each test the vibrating arm is applied very lightly parallel to the skin of the area to be tested. The patient states whether or not he feels a momentary sense of vibration.

Scoring. For each site where vibration is felt, 1 point. Thus, for each tuning fork test, the maximum score for one nerve is 3 points.

Note. This technique differs from the usual method of testing for vibration sensitivity, in which the base of the tuning fork is pressed firmly on the skin and elicits deep sensation. In the test as described above, the vibration of the moving arm of the tuning fork is rapidly damped, however lightly the skin is touched. However, during a brief period (maybe $0.5 \mathrm{~s}$ ) when vibration continues, a very distinctive superficial 'buzzing' sensation is felt, due to stimulation of the rapidly acting mechano-receptors.

Pin prick test

The pin/needle used for this test must be sterile and not too sharp; a slightly blunted surgical or hypodermic needle is suitable. The point should be lightly touched on the skin using about $5 \mathrm{~g}$ force; the test must not cause bleeding. The patient, if he feels the stimulus, states if it is sharp or blunt compared with the sensation when normally sensitive skin is touched in the same way. The result is recorded for each test site as in Table 6. The maximum possible score for one nerve is 9 points. 
Table 6. Scoring for pin-prick test

\begin{tabular}{cc}
\hline Sensation elicited & Score \\
\hline Sharp & 3 \\
Blunt & 0 \\
Nil & 0 \\
\hline
\end{tabular}

Thermoreceptors

No specific technique is suggested; hot or cold applicators must be of constant and known temperature and be applied for a standard time.

\section{Voluntary muscle power tests}

These are performed by standard methods, on selected muscles:

Ulnar (Superficial branch) - Abductor Digiti Minimi

Ulnar (deep branch) $\quad-1$ st dorsal interosseous

Median - Abductor Pollicis Brevis

The grading system is the Medical Research Council scale, adapted for small muscles (Table 7).

Scoring

Ulnar - VMT score Abductor Digiti Minimi + VMT score 1st dorsal interosseous.

Median - VMT score Abductor Pollicus Brevis $\times 2$.

Thus the maximum score for one nerve is 10 points.

Table 7. Scoring system for voluntary muscle power tests

\begin{tabular}{clc}
\hline Grade & \multicolumn{1}{c}{ Definition* } & Score \\
\hline 5 & Normal range of movement, normal power & 5 \\
4 & Normal range of movement, less than normal power & 4 \\
3 & Normal range of movement against slight or no resistance & 3 \\
2 & Reduced range of movement when there is no resistance & 2 \\
1 & Muscle flicker, no (or minimal) joint movement & 1 \\
0 & Complete paralysis & 0 \\
\hline
\end{tabular}

*If there is restriction of passive movement, 'normal range' is taken to be the range of passive movement.

\section{Nerve conduction velocity}

The stimulating points (Table 8) are marked on the skin, and stimuli delivered that are sufficiently strong to elicit a maximal response from the recording electrodes which are sited in abductor digiti minimi (ulnar nerve) and abductor pollicis brevis (median nerve). The length of each nerve segment is measured, and the conduction velocity of the fastest motor fibres for each nerve segment 
Table 8. Stimulating points for nerve conduction velocity tests

\begin{tabular}{ccc}
\hline Stimulating point & Ulnar nerve & Median nerve \\
\hline 1 & $\begin{array}{c}\text { Wrist } 5 \text { cm proximal to the } \\
\text { recording electrode } \\
4 \text { cm distal to the medial epi- } \\
\text { condyle of the humerus }\end{array}$ & $\begin{array}{c}\text { Wrist } 6 \mathrm{~cm} \text { proximal to the } \\
\text { recording electrodes } \\
2 \mathrm{~cm} \text { distal to the most } \\
\text { distal elbow crease }\end{array}$ \\
3 & $\begin{array}{c}\text { cm proximal to the medial } \\
\text { epicondyle of the humerus }\end{array}$ & In the axilla \\
4 & In the axilla & \\
\hline
\end{tabular}

Table 9. Scoring of nerve conduction velocity tests

\begin{tabular}{cr}
\hline $\begin{array}{c}\text { Conduction velocity } \\
(\text { metres/sec) }\end{array}$ & Points \\
\hline $75-55$ & 10 \\
$54-45$ & 8 \\
$44-35$ & 6 \\
$34-25$ & 4 \\
$24-5$ & 2 \\
$4-0$ & 0 \\
\hline
\end{tabular}

and for the whole length of the nerve determined. Correction to standard temperature is theoretically desirable, but in practice it seldom alters the grading of a nerve. The method of scoring is shown in Table 9: scores are allotted for the whole nerve and also to the most affected (slowest conducting) segment of each nerve. The sum of these scores (maximum is 20) is the nerve conduction velocity score for each nerve.

Additional information that could be optionally obtained includes:

1 The area of the action potential (the integral of the curve) which is proportional to the number of working motor units. When an integrator is not available the curve can be copied on paper, cut out and weighed.

2 The time interval between the start and the peak of the evoked action potentials.

\section{Assessment of nerve pain}

The presence and severity of pain will be recorded according to the definitions shown in Table 10. The maximum score for each nerve is 3 points.

\section{Assessment of tenderness}

Tenderness is present if the patient winces when the nerve is palpated. It will be recorded and graded according to the definitions shown in Table 11. The maximum score for each nerve is 3 points. 
Table 10. Grading and scoring system for severity of nerve pain

\begin{tabular}{lc}
\hline \multicolumn{1}{c}{ Grade } & Score \\
\hline Absent & 3 \\
Mild (Only aware intermittently and does not & 2 \\
$\quad$ limit activity) & \\
Moderate (Sleep disturbed, activities diminished, & 1 \\
$\quad$ work efficiency diminished) & 0 \\
\hline
\end{tabular}

Table 11. Grading and scoring system for severity of nerve tenderness. (If tenderness is present at more than one site of a nerve, the most tender site should be scored.)

\begin{tabular}{lc}
\hline \multicolumn{1}{c}{ Grade } & Score \\
\hline Absent & 3 \\
Mild (Absent if patients' attention is distracted) & 2 \\
Moderate (Present if attention is distracted) & 1 \\
Severe (Very tender and patient withdraws the arm & \\
$\quad$ forcibly) & 0 \\
\hline
\end{tabular}

Table 12. Grading and scoring system for stretch test

\begin{tabular}{lc}
\hline Grade & Score \\
\hline $\begin{array}{l}\text { Negative No pain even on full passive elbow flexion } \\
\text { Mild No pain on full active elbow flexion; pain only } \\
\text { when elbow is passively flexed further }\end{array}$ & 3 \\
$\begin{array}{l}\text { Moderate Pain on active flexion of the elbow, but can } \\
\text { be flexed up to or even beyond } 90^{\circ}\end{array}$ & 2 \\
Severe Cannot flex elbow up to $90^{\circ}$ because of pain & 1 \\
\hline
\end{tabular}

\section{Stretch test and compression tests}

If positive these tests may indicate that the ulnar nerve has become shortened and tight during inflammation, or they may indicate the possible presence of sites of entrapment.

\section{Stretch Test}

First, fully dorsiflex the wrist with the elbow fully extended, then slowly flex the elbow. Record the angle at which elbow flexion is restricted by pain in the ulnar nerve at the elbow.

Compression Test

With the elbow extended, flex all fingers and dig the fingers into the palm. Then flex and ulnar deviate the wrist. Pain is felt in the ulnar nerve above and/or below the elbow. 
Table 13. Grading and scoring system for compression test

\begin{tabular}{lc}
\hline \multicolumn{1}{c}{ Grade } & Score \\
\hline Negative (No pain) & 3 \\
Mild Pain present. Wrist and finger position can be & 2 \\
$\quad$ maintained & \\
Moderate Pain prevents wrist and finger position from & 1 \\
$\quad$ being maintained & 0 \\
\hline
\end{tabular}

\section{Scoring}

The grading and scoring systems for these tests are shown in Tables 12 and 13 . For each test the maximum score for a nerve is 3 points.

\section{Acknowledgements}

The protocol was prepared by a workshop held at the Schieffelin Leprosy Research and Training Centre, Karigiri, 12-14 March, 1980; subsequent minor modifications were agreed by correspondence. The members of the workshop were:

Dr J Andersen (Ethiopia), Dr M Gregor Anderson (USA), Dr N H Antia (India), Dr Berbudi (Indonesia), Dr P W Brand (USA), Dr F Duerksen (Paraguay), Dr E P Fritschi (India), Dr J Hargrave (Australia), Dr J H James (Kenya), Professor C K Job (India), Dr R Kazen (Sierra Leone), Mr S L Kolumban (India), Dr B Naafs (Netherlands), Dr D D Palande (India), Dr V K Pannikar (India), Dr J M H Pearson (Chairman; India), Dr D J Pring (Secretary; India), Dr M S Nilakanta Rao (India), Professor A J Selvapandian (India), Dr H Srinivasan (India), Dr Phyllis M Taylor (India), Dr Julia K Terzis (Canada), Dr R H Thangaraj (India), Dr (Mrs) E S Thangaraj (India), Dr Grace Warren (Thailand).

The participants wish to thank the Leprosy Mission (International), the American Leprosy Mission, the Netherlands Leprosy Relief Organization and the All Africa Leprosy and Rehabilitation Training Centre (ALERT) for their generous support of the Workshop, together with the many staff members of the Schieffelin Leprosy Research and Training Centre who contributed to the planning and organization of the meeting.

\section{Appendix}

METHOD OF FABRICATING STIMULATORS FOR TESTING FOR TOUCH THRESHOLD

A bicycle spoke or similar length of thick wire forms a suitable handle. The nylon thread may be attached to it by plastic bands - the insulation of electrical flex has proved satisfactory for this purpose. 
The tip of each nylon thread should be rounded by passing it momentarily (about $0.25 \mathrm{~s}$ ) through the flame of a spirit lamp. This procedure will remove the sharp irregularities from the cut tip, without forming a fused nylon blob.

Calibration of the threads is most readily achieved if they are already slightly bent. They should be pressed on the pan of a suitable balance so that they bend slightly more. The lengths of the threads are adjusted so that they bend when forces of $0.5 \mathrm{~g}, 2.0 \mathrm{~g}, 5.0 \mathrm{~g}$ and $10.0 \mathrm{~g}$, respectively are applied. 